\title{
Myocardial perforation by flexible Elema endocardial pacing catheters ${ }^{1}$
}

\author{
B A R R IE J. HURWITZ, MONTYM. ZION, \\ and I. W. PROMUND OBEL \\ Department of Medicine, University of the Witwatersrand, and Cardiac Clinic, \\ Johannesburg General Hospital, Johannesburg, South Africa
}

\begin{abstract}
Hurwitz, B. J., Zion, M. M., and Promund Obel, I. W. (1974). Thorax, 29, 678-684. Myocardial perforation by flexible Elema endocardial pacing catheters. Myocardial perforation as a complication of permanent endocardial pacing with flexible Elema catheters is described in nine patients. In eight patients pacing failed, but there were no other serious ill-effects. Diaphragmatic pacing occurred in five patients. Radiological evidence of movement, either posteriorly and/or laterally, of the electrode tip was detected in five patients. In only two of them was the electrode seen to be definitely extracardiac in position. A change in the pacing wave form aided the diagnosis in two patients. In a further two patients electrode tip electrocardiograms helped to confirm the diagnosis where, upon withdrawal of the perforating electrode, small $Q$ waves disappeared, RS complexes enlarged, and the ST segments became more elevated, with deeply inverted $\mathrm{T}$ waves.

Withdrawal and repositioning of the electrode catheters was performed in seven patients. In one, a new catheter was inserted, and in another, where uninterrupted cardiac pacing was accompanied by intermittent diaphragmatic pacing, the pacing system was left unaltered. The follow-up is from six months to four and a half years with one late death apparently unrelated to pacing failure.
\end{abstract}

Perforation of the myocardium by endocardial catheters is reported to occur in up to $10 \%$ of patients (Meyer and Millar, 1968). This complication has been related to the stiffness and thickness of the catheters and is one of the reasons why later models are being made thinner and more flexible (Thalen, van den Berg, van der Heide, and Nieveen, 1969a). The incidence of perforation with modern catheters is low, only 61 patients having been reported in the literature.

Three hundred and ten patients from our clinic have been paced with permanent flexible Elema ${ }^{2}$ endocardial catheters. We have recognized nine instances of right ventricular perforation in this group. Although use of this catheter has been described in a number of large series (Lagergren et al., 1966; Edhag, 1969), there have been no previous reports of perforation with it.

\section{PATIENTS}

The nine patients were aged 66 to 83 years with an average of 75 years. There were eight women and

1Supported in part by the Medical Research Council of South Africa 'Elema Schönander, Solna, Sweden

one man. All were paced because of Adams-Stokes attacks due to various types of heart block. Evidence of ischaemic heart disease was present in all, in the form of either well-documented myocardial infarctionx or persistent angina pectoris. All had cardiomegaly. The pacing thresholds at the time of catheter inser tion, and elapsed time to perforation are summarized in Table $\mathrm{I}$.

\section{METHODS OF PACING}

In all instances an Elema 588B pacing catheter was used. This catheter is an extremely soft flexible cable․ㅡㄹ.

\begin{tabular}{|c|c|c|c|}
\hline \multicolumn{4}{|c|}{ PATA } \\
\hline Patient & Age/Sex & Initial Pacing Threshold & $\begin{array}{c}\text { Time to } \\
\text { Perforation }\end{array}$ \\
\hline $\begin{array}{l}1 \\
2 \\
3 \\
4 \\
5 \\
6 \\
7 \\
8 \\
9\end{array}$ & $\begin{array}{ll}77 & F \\
66 & F \\
80 & F \\
80 & F \\
75 & \mathbf{M} \\
72 & F \\
80 & F \\
66 & F \\
83 & F\end{array}$ & $\begin{array}{l}\text { ND } \\
\text { ND } \\
2 \cdot 0 \mathrm{~V} \\
1 \cdot 3 \mathrm{~V} \\
\text { ND } \\
2 \cdot 0 \mathrm{~V} \\
1 \cdot 8 \mathrm{~V} \\
1 \cdot 2 \mathrm{~V} \\
3 \cdot 6 \mathrm{~V}\end{array}$ & $\begin{array}{l}6 \text { days } \\
24 \text { days } \\
7 \text { days } \\
10 \text { months } \\
8 \text { months } \\
26 \text { months } \\
3 \text { days } \\
2 \text { days } \\
3 \text { days }\end{array}$ \\
\hline
\end{tabular}


$1.2 \mathrm{~m}$ long and $1.2 \mathrm{~mm}$ in diameter. It consists of a spun textile core around which four thin strips of stainless steel have been wound. There is an insulated covering of polyethylene. The electrode tip is a cylindrical platinum knob, $2.5 \mathrm{~mm}$ in diameter and $10 \mathrm{~mm}$ long, partly covered by a small piece of silicone rubber to prevent blood from entering the connection between cable and tip.

The catheter was inserted via the exposed right external jugular vein and the electrode tip was manipulated into position in the right ventricular apex under fluoroscopic control. The technique used was to float the catheter into the ventricle with the patient lying on his left side, as described previously (Zion et al., 1964). A stiffening cannula was never used. Postero-anterior and lateral chest radiographs were taken and the pacing threshold was identified at the conclusion of the procedure. The distal end of the catheter was left exteriorized for three to five days to facilitate further manipulation in the event of early displacement. When the electrode position had been shown to be stable and the pacing threshold remained at an acceptable level, the catheter was tunnelled subcutaneously to the right iliac fossa and attached to an implanted power source.

\section{DIAGNOSIS OF PERFORATION}

Myocardial perforation was diagnosed by clinical, radiological, electronic, and electrocardiographic criteria (Table II).

T A B L E I I

DIAGNOSTIC FEATURES OF PERFORATION

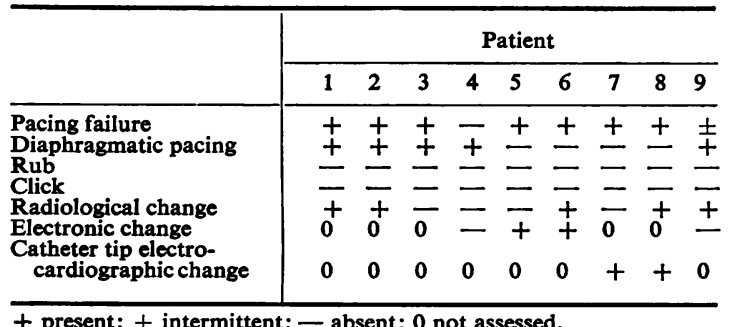

Clinical Changes Pacing failed completely in seven patients, and one patient (9) paced intermittently. None of the patients experienced Adams-Stokes attacks following pacing failure but four developed 'giddiness'.

Diaphragmatic pacing occurred in five patients. In one instance (4), this was intermittent, being most marked in full expiration with the patient in either the erect or prone positions, and was not associated with failed cardiac pacing. The diagnostic features in this case have been reported separately (Hurwitz and Obel, 1973).

${ }^{1}$ (Type 561) Tektronix Inc., Portland, Oregon, USA
Friction rubs and extracardiac clicks were never heard.

Radiological CHANGes Postero-anterior and lateral chest radiographs were obtained in each patient. In five, the electrode tip had changed its position, but in only two was a definite extracardiac position visualized. In three patients the electrode tip appeared merely to have advanced more laterally and posteriorly. The remaining four showed no detectable change in electrode position.

ELECTRONIC CHANGES The rate of discharge and wave form of the implanted pacing unit was measured in each of the three standard limb leads on a calibrated rapid sweep oscilloscope ${ }^{1}$ in patients $4,5,6$, and 9 . No changes were detected in two patients ( 4 and 9 ), when compared to previous records. At this time cardiac pacing was uninterrupted in patient 4 and intermittent in patient 9. In patients 5 and 6 the amplitude dropped considerably, and the artefact assumed a square wave configuration. There was no change in the pulse duration or rate of pacemaker discharge. Because of this unusual configuration, together with no obvious change in catheter tip position in patient 5 , a faulty pacing unit or faulty connection between catheter and pacing unit was suspected. The power unit was then electively replaced, but the system still failed to pace and measurements showed the same altered wave form in terms of size and configuration (Fig. 1). A new pacing catheter was then inserted and connected to this unit, following which cardiac pacing was immediate and the pulse wave reverted to its

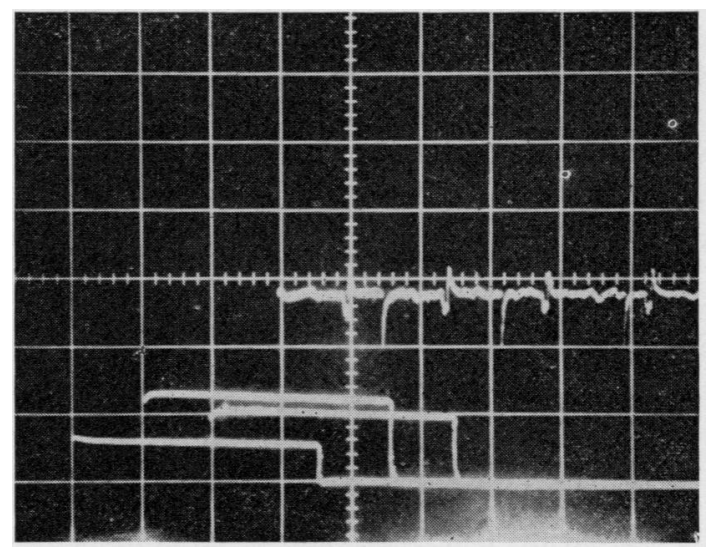

FIG. 1. Patient 5 during perforation. Pacing artefact wave forms recorded in standard leads 1,2 , and 3 . 
initial (preperforation) configuration (Fig. 2). The original power unit appeared to be functioning normally on subsequent electronic testing. A similar change in pulse wave configuration occurred in patient 6 together with radiological evidence of electrode perforation.

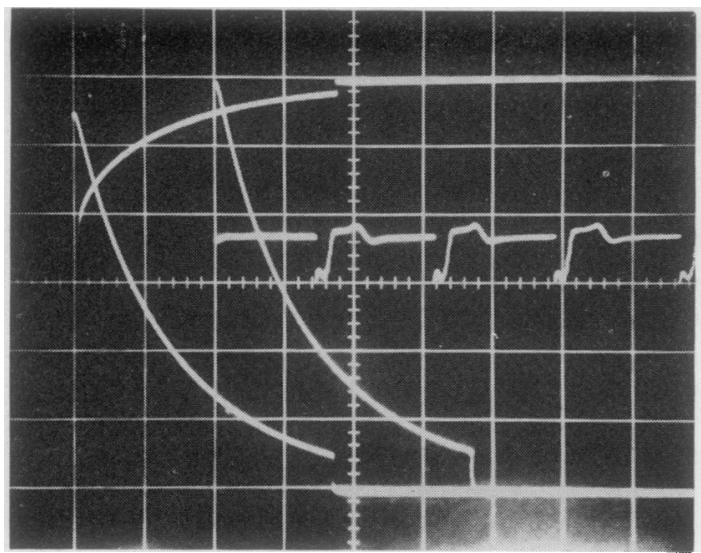

FIG. 2. Patient 5 with new catheter. Pacing artefact wave forms in standard leads 1, 2, and 3. Polarity is reversed in standard lead 3.

In order to test the hypothesis that these electronic changes in the pacing artefact were due to perforation, measurements were made with the pacing unit implanted in the subcutaneous tissues of the abdomen, attached at one time to an intracardiac electrode, and at another to an identical electrode inserted into the subcutaneous tissue at the level of the xiphisternum. In the former situation a normal wave form was present, while in the latter situation a square wave configuration of low amplitude occurred together with no change in the pulse duration (Fig. 3).

ELECTROCARDIOGRAPHIC CHANGES When pacing failed in two patients ( 7 and 8 ) soon after electrode insertion ( 3 and 2 days respectively), the distal end of the pacing catheters was still exteriorized and thus electrode tip electrocardiographic recordings could be made. The presenting signs of perforation in both were rapidly rising pacing thresholds and failed pacing, in spite of the use of an external pacemaker ${ }^{1}$, which has an output of up to 30 milliamps. The electrode tip electrocardiogram in patient 7 , at the time of failed pacing (Fig. 4a), showed a QRS pattern with slightly elevated ST segments and inverted T

\footnotetext{
${ }^{1}$ Medtronic Model 5880C
}

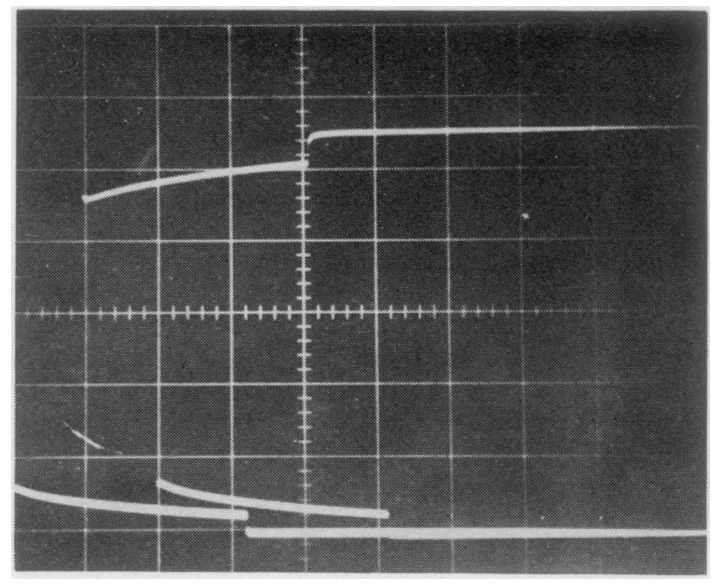

FIG. 3. Pacing artefact wave forms in an example of simulated perforation. Standard leads 1, 2, and 3 . Polarity is reversed in standard lead 3.

waves. After the catheter had been withdrawn $2 \mathrm{~cm}$ the electrode tip electrocardiogram (Fig. 4b) showed larger RS complexes and disappearance of the $Q$ waves. The ST segments became more elevated and the $\mathrm{T}$ waves more deeply inverted. Pacing recommenced at a threshold of 3.0 volts. In patient 8 the electrode had advanced inferiorly, apparently beyond the heart border, when pacing failed and the electrode tip electrocardiogram (Fig. 5a) showed similar recordings to those found in patient 7 (Fig. 4a). As the catheter was withdrawn into the right ventricle, electrode tip electrocardiogram recordings showed enlargement of the RS complexes together with disappearance of the $Q$ waves. The ST segments varied and then $\stackrel{0}{x}$ became elevated (Fig. 5b). In the final position at the apex of the right ventricle, the electrode tip electrocardiogram (Fig. 5c) was similar to that of patient 7 (Fig. 4b).

TREATMENT AND FOLLOW-UP

Repositioning was performed in seven patients without any immediate complication. In one (1), $\sigma$ subsequent repeated electrode displacement (with- $N$ out further perforation) led to the use of epicar- N dial leads 13 months later. Wound sepsis necessi- $\omega$ tated revision of the entire pacing system in patient 3. In patient 5 the perforated catheter was $\stackrel{0}{=}$ so firmly attached to the heart wall that retraction $\stackrel{\infty}{\rightarrow}$ of it displaced the entire heart upwards; further attempts at retraction were thus abandoned and 0 another Elema catheter was inserted. Patient $7 \overrightarrow{\mathbb{D}}$ developed a post-pericardiotomy syndrome 20 days $\stackrel{?}{\circ}$ after repositioning, which responded to ibuprofen. 
FIG. 4. Patient 7. Unipolar electrocardiograms from pacing catheter (see text): (a) at perforation; (b) upon catheter withdrawal.

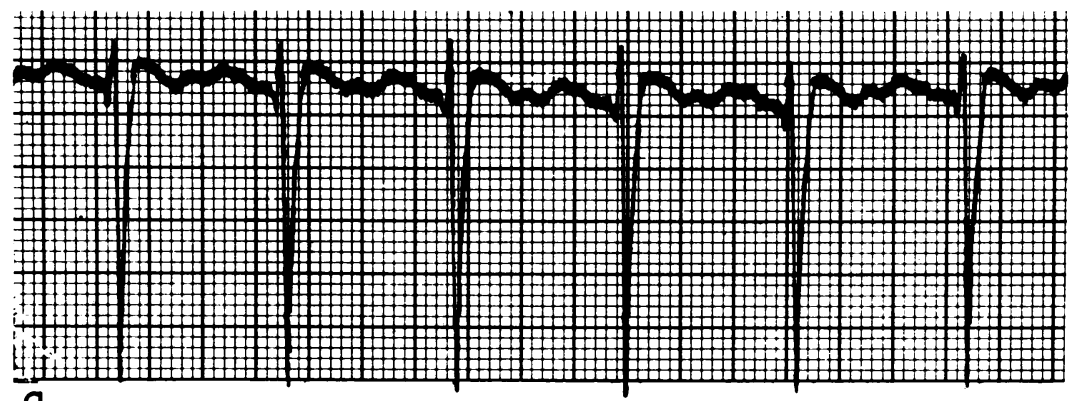

a.
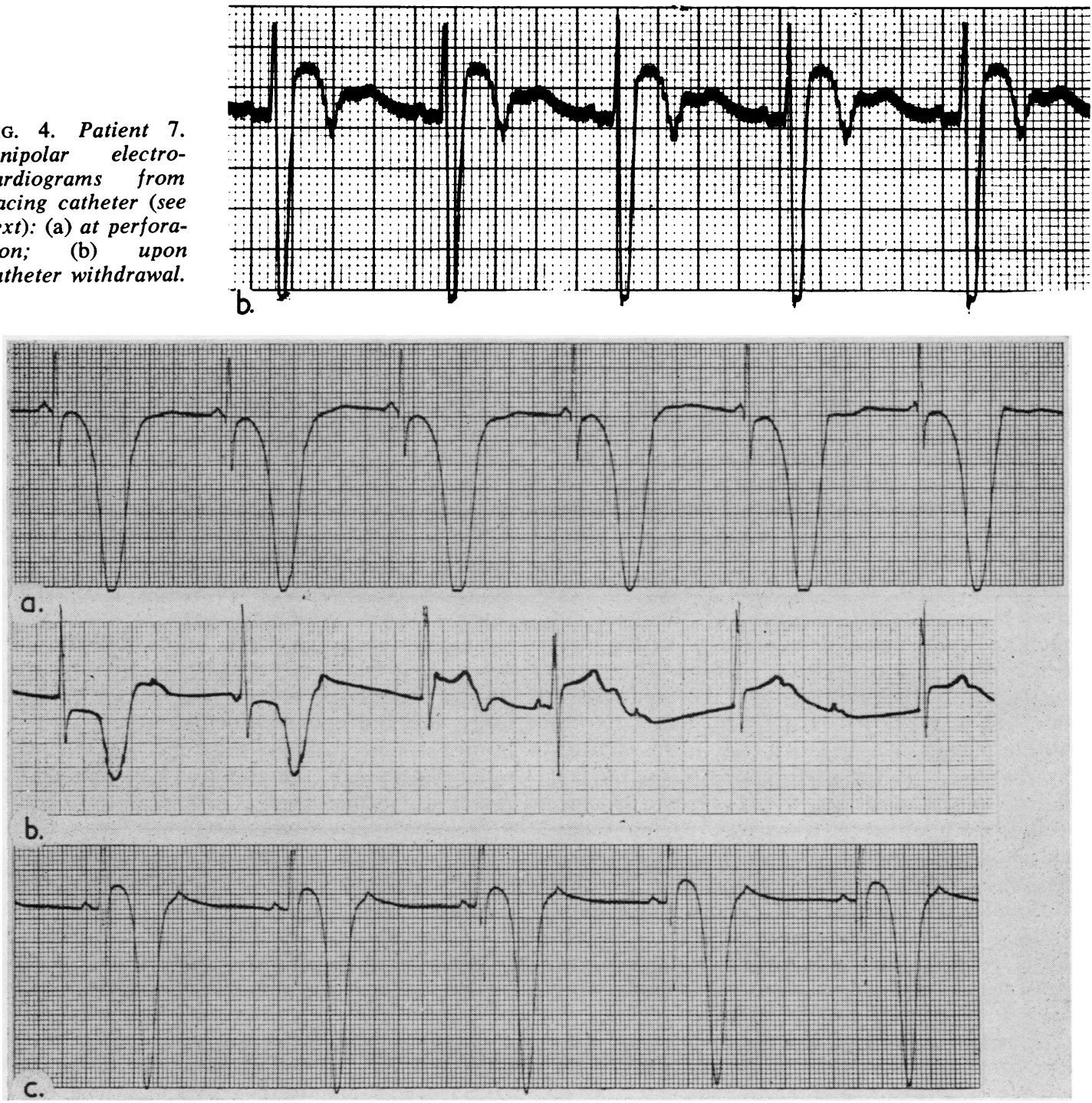

FIG. 5. Patient 8. Unipolar electrocardiograms from pacing catheter (see text): (a) at perforation; (b) during withdrawal; (c) upon withdrawal and repositioning. 
In patient 4 the catheter was not repositioned as cardiac pacing was uninterrupted and the electrode had been stable in position over a prolonged period of time.

The follow-up to date is from six months to four and a half years. Patient 4 died 19 months after perforation of a probably myocardial infarct; cardiac pacing had been uninterrupted until death. A necropsy was not performed. There have been no other deaths.

\section{DISCUSSION}

Perforation of the heart is uncommon with permanent flexible endocardial pacing catheters. Of the 61 recorded instances (Meyer and Millar, 1968; Barold and Center, 1969; Furman, Escher, and Solomon, 1969; Sykosch, 1969; Goswami, Gould, Gomprecht, and Imperiale, 1971; Bernstein, Rotem, and Peretz, 1971) most have related to the use of either Medtronic or Cordis catheters, some of which have been left with a stylet in situ and all of which have been positioned with a stylet. Although the use of the flexible Elema catheter (588B) has been described in several large series (Lagergren et al., 1966; Schaudig, Thurmayr, and Zenker, 1971), we have been unable to find reports of perforation by this catheter and the manufacturers, Elema Schönander, confirm its apparent safety, although displacement (some of which may actually be perforation) is not uncommon (Grant et al., 1973). On the other hand, the diagnosis of perforation may present difficulties.

Absolute proof of myocardial perforation requires either surgical exposure of the heart or undoubted radiological demonstration of the electrode lying in an extracardiac position. This proof is usually not obtainable before the diagnosis is made, as surgery is seldom indicated either to institute pacing or to repair a tear in the myocardium produced by the perforation. In the clinical context, intermittent or failed cardiac pacing, together with either extracardiac pacing (chest wall, abdominal wall or diaphragm), a pericardial rub, or radiological shifts in catheter position usually signifies cardiac perforation. Verification of this diagnosis, and retrospective proof, is obtained when cardiac pacing recommences and extra-cardiac pacing ceases upon electrode withdrawal into the cavity of the right ventricle.

We believe exit block has been excluded as the cause of pacing failure in our nine patients by the presence of at least one other diagnostic feature of perforation besides failed pacing (Table II).

Diaphragmatic pacing occurred in five of our patients and was associated with failed cardiac pacing in four of them. Withdrawal of the elec-흠 trode tip into the heart re-established cardiac $\frac{\rho}{\sigma}$ pacing in four $(1,2,3$, and 9). Patient 4 was尺 unique in that uninterrupted cardiac pacing continued with intermittent diaphragmatic pacing. $\vec{\circ}$

Only five of our patients demonstrated radiological change in electrode position, and in only $\vec{\omega}$ two did the electrode appear to be clearly extracardiac in situation. Our attempts to utilize the technique of Ormond, Rubenfire, Anbe, andin Drake (1971), who reported better diagnosis with close visualization of the epicardial fat pad line, were unsuccessful in patients 6,7 , and 8 . Pre- $-\infty$ sumably the diagnostic value of radiology in? perforation is limited by the fact that the rightventricular myocardium is only 4 to $5 \mathrm{~mm}$ thick, $Z$ and penetration of this wall may occur with little or no obvious radiological change in electrode 3 position.

A change in the electrical axis of the pacing $\vec{\oplus}$ stimulus, pericardial friction rubs, and extracardiac auscultatory clicks (Kramer, Moss, and Shah, 1970) may also suggest the diagnosis, but these signs were not helpful in our experience.

Changes in the shape and size of the pacingo artefact in patients 5 and 6 were useful as diag-0 nostic pointers. Similar changes were found in ao case of simulated perforation, and although such $\overrightarrow{\bar{\sigma}}$ changes may occur with fractured catheters or 3 poor connections (Thalen, van den Berg, van der? Heide, and Nieveen, 1969b), there was no evi-0. dence of either of these in our cases. We have not seen such pulse wave changes from othero causes of failed pacing.

Changes in the electrode tip electrocardiogramo occurred in patients 7 and 8 and, as reported by Barold and Center (1969), may be useful in diag-o̊ nosis, but the opportunity of obtaining them is limited as perforation usually occurs after implan-윽 tation. Although the elevated ST segment can $\square$ revert to normal over a period of time without myocardial perforation (Gordon, Vagueiro, andn Barold, 1968), a decrease in size of QRS voltage is most unlikely. The initial $Q$ wave found in patients 7 and 8 at the time of perforation wasw subsequently replaced by an RS complex upong withdrawal of the electrodes into the right ventrito cle. In left anterior hemiblock and right bundle-⿱ branch block (present in both) as with normal ${ }^{?}$ conduction, left ventricular leads show initial $Q^{O}$ waves. Direct left ventricular leads thus show aO QRS pattern and right ventricular leads an RSR pattern (Goldman, 1970). We have interpreted the $\mathbb{\Omega}_{\mathcal{Q}}$ presence of $Q$ waves in our patients as being duego 
to the perforated electrodes recording left ventricular complexes from the pericardial space.

The mechanism of perforation with the use of these extremely flexible catheters is purely speculative. The distinct time lapse (in three patients more than six months) between insertion of the electrode and perforation, as well as the fact that stylets or stiffeners were never used, argue strongly against the method of insertion being responsible. We can find no evidence to support the contention that decreasing heart size or increasing contractility with pacing cause perforation (Sykosch 1969; Goswami et al., 1971). The postulate that a rigid electrode tip may be forced through the ventricular wall by constant apposition of ventricular myocardium in a cul-de-sac formed by the intraventricular septum and apex and free wall of the right ventricle is suggested by the frequency with which the perforated electrodes point towards the apex of the heart in the postero-anterior radiograph but posteriorly in the lateral view.

Some of the clinical features of our nine patients are somewhat at variance with those usually found in patients paced in Johannesburg (Obel, Marchand, and Scott Millar, 1972) and may thus indicate aetiological factors. In particular, they are older and have a female preponderance. Ischaemic heart disease was present in all nine patients, whereas it was present in only $29 \%$ of other patients paced over the same period.

The most important complication of myocardial perforation is failed pacing. Indeed, this is usually the presenting feature. Although this complication is potentially lethal, most cases have been relatively easily managed. Haemorrhage, with subsequent tamponade, rarely occurs. Likewise retraction and repositioning of perforated catheters has not caused tamponade in most authors' experience (Meyer and Millar, 1968; Sykosch, 1969; Bernstein et al., 1971). Therefore we believe that this is a benign and safe procedure. However, if resistance to withdrawal is encountered, the catheter could be left in situ and a new one inserted. In the presence of such resistance, forcible withdrawal probably constitutes a real risk of tearing the myocardium, haemorrhage, and tamponade (Battle, Banas, and Levine, 1973).

\section{REFERENCES}

Barold, S. S. and Center, S. (1969). Electrographic diagnosis of perforation of the heart by pacing catheter electrode. American Journal of Cardiology, 24, 274.
Battle, W. E., Banas, J. S., and Levine, H. J. (1973). Papillary muscle rupture of the mitral valve complicating removal of a permanent transvenous electrode. Chest, $63,455$.

Bernstein, V., Rotem, C. E., and Peretz, D. I. (1971). Permanent pacemakers. 8 year follow-up study. Annals of Internal Medicine, 74, 361.

Edhag, O. (1969). Long-term cardiac pacing. Experience of fixed-rate pacing with an endocardial electrode in 260 patients. Acta Medica Scandinavica, 186 Supplement, 502, p. 36.

Furman, S., Escher, D. J. W., and Solomon, N. (1969). Experiences with myocardial and transvenous implanted cardiac pacemakers. American Journal of Cardiology, 23, 66.

Goldman, M. J. (1970). Principles of Clinical Electrocardiography. 7 th ed. p. 123. Lange, Los Altos.

Gordon, A. J., Vagueiro, M. C., and Barold, S. S. (1968). Endocardial electrograms from pacemaker catheters. Circulation, 38, 82.

Goswami, M., Gould, L., Gomprecht, R. F., and Imperiale, A. (1971). Perforation of the heart by flexible transvenous pacemaker. Journal of the American Medical Association, 216, 2013.

Grant, A. F., Jones, D., Hunyor, S. N., and Coles, D. M. (1973). Implantation of permanent cardiac pacemakers. Medical Journal of A ustralia, 2, 257.

Hurwitz, B. J. and Obel, I. W. P. (1973). Longstanding cardiac perforation by a permanent endocardial pacing electrode. Chest, 64, 366.

Kramer, D. H., Moss, A. J., and Shah, P. M. (1970). Mechanisms and significance of pacemakerinduced extracardiac sound. American Journal of Cardiology, 25, 367.

Lagergren, H., Johansson, L., Schüller, H., Kugelberg, J., Bojs, G., Alestig, K., Linder, E., Borst, H. G., Schaudig, A., Giebel, O.,, Harms, H.. Rodewald, G., and Scheppokat, K. D. (1966). 305 cases of permanent intravenous pacemaker treatment for Adams-Stokes Syndrome. Surgery, 59, 494.

Meyer, J. A. and Millar, K. (1968). Perforation of the right ventricle by electrode catheters: a review and report of 9 cases. Annals of Surgery, 168, 1048.

Obel, I. W. P., Marchand, P., and Scott Millar, R. N. (1972), Cardiac pacemaking: Experience with the first 120 patients treated in Johannesburg. South A frican Medical Journal, 46, 2002.

Ormond, R. S., Rubenfire, M., Anbe, D. T., and Drake, E. H. (1971). Radiographic demonstration of myocardial penetration by permanent endocardial pacemekers. Radiology, 98, 35.

Schaudig, A., Thurmayr, R., and Zenker, R. (1971). Results of transvenous pacing. Journal of Cardiovascular Surgery, 12, 281.

Sykosch, H. J. (1969). Special problem in cardiac stimulation: A review on 500 pacemaker patients. Geriatrics, 24, No. 12, 78. 
Thalen, H. J. Th., van den Berg, J. W., van der Heide, J. N. H., and Nieveen, J. (1969a). The Artificial Cardiac Pacemaker, p. 90. Royal Vangorcum, Assen, Netherlands.

(1969b). The artificial Cardiac Pacemaker, p. 205 and p. 290. Royal Vangorcum, Assen, Netherlands.

Zion, M. M., Obel, I. W. P., Marchand, P., Fuller,
D. N., and Barlow, J. B. (1964). Electrical pacing of the heart in atrioventricular block. South African Medical Journal, 38, 781.

Requests for reprints to: I. W. P. Obel, Department $\stackrel{\Phi}{\triangle}$ of Medicine, University of the Witwatersrand, Cardiac Clinic, Johannesburg General Hospital, Johannesburg 2001, South Africa. 